УДК 541.183

\title{
ГРАФОАНАЛИТИЧЕСКАЯ ОЦЕНКА ДИНАМИЧЕСКОЙ ЕМКОСТИ ГЛАУКОНИТА ПРИ СОВМЕСТНОЙ СОРБЦИИ КАТИОНОВ Сu(II), $\mathrm{Ni}(\mathrm{II}), \mathrm{Zn}(\mathrm{II})$
}

\author{
(C) 2018 В. И. Вигдорович ${ }^{1,2}$, Т. В. Жуковская ${ }^{2}$ Л. Е. Цыганкова ${ }^{3}$, М. Н. Урядникова ${ }^{3}$, Н. В. Шель ${ }^{2}$ \\ ${ }^{1}$ Всероссийский научно-исследовательский институт использования техники и нефтепродуктов \\ в сельском хозяйстве», Ново-Рубежный пер., 28, 392022 Тамбов, Россия \\ ${ }^{2}$ Тамбовский государственный технический университет, ул. Советская, 106, 392000 Тамбов, Россия \\ ${ }^{3}$ Тамбовский государственный университет имени Г. Р. Державина, \\ ул. Интернаџиональная, 33, 392000 Тамбов, Россия \\ e-mail:vits21@mail.ru
}

Поступила в редакцию 02.10.2017

\begin{abstract}
Аннотация. Предложен расчетный способ оценки количества вещества адсорбата $(N)$ и динамической емкости сорбента $(Q)$ в условиях очистки от загрязнителей в потоке. Рассмотренные подходы действительны при использовании сорбентов и удалении сорбатов любой природы из проточных сред независимо от скорости потока элюата в условиях ламинарного течения жидкости.

Способ основан на использовании $\beta$-сплайновых кривых и базируется на свойствах геометрической непрерывности, используемых при решении задач геометрического моделирования посредством кубических многочленов. Он позволяет:

- рассчитать величины динамической емкости $Q$ к любому моменту времени от начала сорбции при отсутствии экспериментальной оценки величины коэффициента сорбции $\rho_{\tau_{i}}$;

- оценить рассчитанное время непрерывной работы сорбента до его замены или регенерации.

При комнатной температуре получены величины $Q_{\mathrm{i}}$ глауконита по катионам $\mathrm{Cu}(\mathrm{II}), \mathrm{Ni}(\mathrm{II}), \mathrm{Zn}$ (II) при их совместной сорбции как функция природы адсорбатас учетом высоты его слоя, линейной скорости подачи растворапри произвольном интервале времени от начала процесса. Оценена относительная сорбционная способность катионов в зависимости от тех же факторов. Показано, что с ростом скорости подачи раствора увеличивается динамическая емкость сорбента, которая изменяется в ряду:

$$
Q_{\mathrm{Ni}(\mathrm{II})}>Q_{\mathrm{Zn}(\mathrm{II})}>Q_{\mathrm{Cu}(\mathrm{II})}
$$
\end{abstract}

Ключевые слова:глауконит, поток, сорбция, медь, никель, цинк, динамическая емкость, расчет.

DOI

\section{ВВЕДЕНИЕ}

Сорбционная способность природных глинистых минералов [1-20] и биопродуктов [21] систематически исследуется с целью использования их в качестве сорбентов для очистки питьевых, технологических и сточных вод, а также в качестве биоиндикаторов чистоты биосферы [21]. Однако в подавляющем большинстве случаев сорбция поллютантов изучается в статических условиях, позволяющих оценить равновесную глубину процесса, его термодинамические характеристики и наиболее вероятный тип изотермы адсорбции [1-17].Исследо- вания сорбции поллютантов из проточных растворов проводятся значительно реже [18-20], хотя закономерности процесса в том и другом случае существенно различаются.Вместе с тем для практики наиболее важен динамический режим, так как именно он позволяет создать непрерывный технологический процесс.

Целью настоящей статьи является изложение разработанного авторами общего метода расчета динамической емкости сорбента независимо от вида кинетической кривой $\rho=f(\tau)$, приведенной на рис. 1 (кривая 2). 
Ранее [22] нами был рассмотрен метод расчета количества вещества поллютанта и удельной динамической емкости сорбента в частном случае, соответствующем кинетической кривой $\rho=f(\tau)$, изображенной на рис. 1 (кривая 1). Величина $\rho$ по физическому смыслу представляет собой коэффициент сорбции, равный отношению разности концентраций сорбата в исходом растворе $C_{0}$ и в заданный текущий момент времени от начала сорбции $\tau_{i}$ к исходной величине:

$$
\rho_{\tau, \mathrm{i}}=\left(C_{0}-C_{\tau, \mathrm{i}}\right) / C_{0} .
$$

По физическому смыслу $\rho_{\tau, i}$ - доля поллютанта, извлеченного адсорбентом из раствора к моменту $\tau_{i}$ от начала процесса.

Кинетическая кривая в самом общем варианте включает три выраженных участка. На участке $\mathrm{AB}$ кинетической кривой имеет место полное сорбционное удаление сорбата. При достижении времени $\tau_{\mathrm{B}}$ начинается его проскок. Линия ZR параллельна оси абсцисс и характеризует величину $\tau_{\ni}$ - время, до которого концентрация поллютанта в выходящем из адсорбера растворе не превышает нормативных требований.

Принципиальная особенность кинетической кривой 1 (рис. 1) в том, что на участке ВМ величина $\rho_{\tau, \mathrm{i}}$ является линейной функцией $\tau_{i}$. Подобная картина наблюдается сравнительно часто [18-20], но все же носит частный характер. Только в этом случае с учетом координат характеристических точек В $(\rho=1)$ и $\mathrm{M}(\rho=0)$ можно получить выражение для участка ВМ кривой 1 (рис. 1) [22] и далее рассчитать количество $(N)$ поглощенного поллютанта и динамическую емкость сорбента $Q$ к моменту времени $\tau_{i}$ на участке ВМ от начала процесса.

На участке MNcopбция отсутствует, так как динамическая емкость насыщена. Тогда суммарные значения $N$ и $Q$ к моменту времени $\tau_{\mathrm{D}}$ находятся суммированием соответствующих значений $N$ и $Q$ участков $\mathrm{AB}$ и $\mathrm{BM}$ :

$$
N_{\Sigma}=N_{\mathrm{AB}}+N_{\mathrm{BM}} \text { и } Q_{\Sigma}=Q_{\mathrm{AB}}+Q_{\mathrm{BM}} .
$$

Весь интервал времени сорбции поллютанта от начала процесса до его завершения $\left(\tau_{\mathrm{M}}\right)$, условно разделен на семь подинтервалов, длительность которых, например, $\Delta \tau=\tau_{\mathrm{C}}-\tau_{\mathrm{B}}$, постоянна.

В подинтервале I (рис. 1) количество сорбированного поллютанта эквивалентно площади $\mathrm{AB} \rho_{\mathrm{B}} \rho_{\mathrm{A}}$, т.е. из раствора в период сорбции $\tau_{\mathrm{A}}-\tau_{\mathrm{B}}$ удален поллютант полностью (в пределах чувствительности анализа).

Количество сорбированного вещества за этот период рассчитывается из зависимости:

$$
N_{\mathrm{AB}}=C_{0} V_{\mathrm{AB}},
$$

где $C_{0}$ - исходная концентрация сорбата, $V_{\mathrm{AB}}-$ объем раствора, прошедшего за этот период через адсорбер.

На участке II количество поглощенного сорбата эквивалентно площади $\mathrm{BC} \rho_{\mathrm{C}} \rho_{\mathrm{B}}$, а оставшегося в вытекшим из адсорбера раствора - площади ВКС, которая заштрихована.На участке III адсорбированное количество вещества эквивалентно пло-

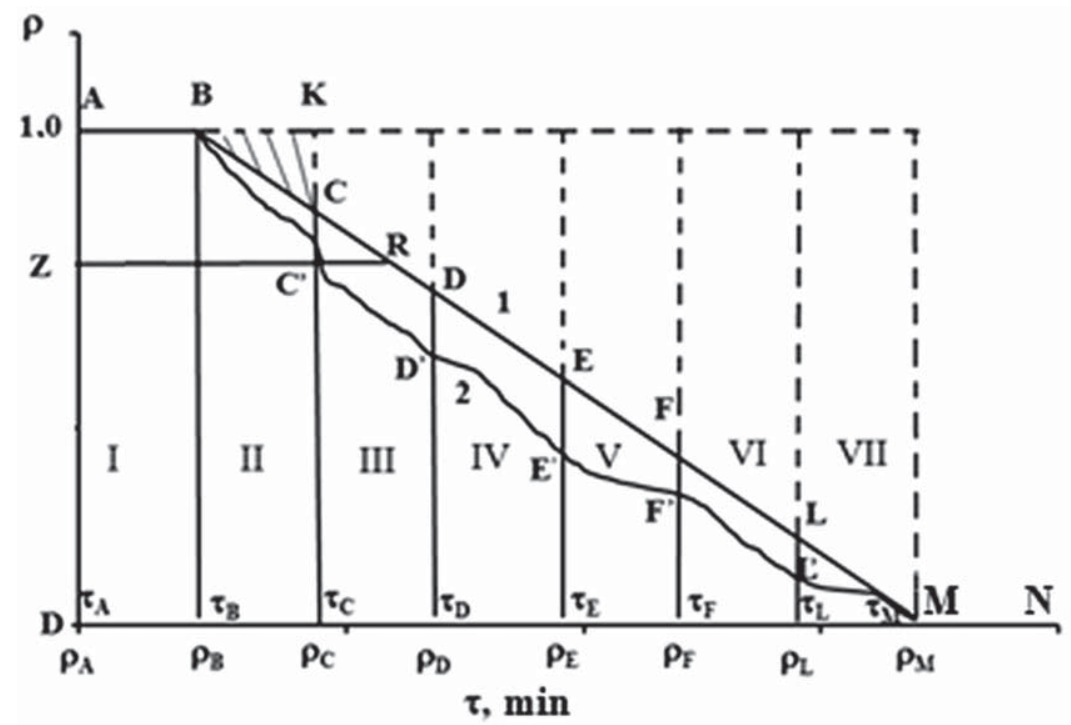

Рис. 1. Схематическое изображение кинетических кривых сорбции с линейной (1) и нелинейной (2) зависимостью $\rho=f(\tau)$ на участке ВМ

[Fig. 1. Schematic representation of the kinetic curves of sorption with linear (1) and nonlinear (2) dependence $\rho=f(\tau)$ in the BM section] 
щади $\mathrm{CD} \rho_{\mathrm{D}} \rho_{\mathrm{C}}$, оставшегося в растворе - разность площадей $\mathrm{AB} \rho_{\mathrm{B}} \rho_{\mathrm{A}}-\mathrm{CD} \rho_{\mathrm{D}} \rho_{\mathrm{C}}$. На остальных участках IV-VII количество вещества сорбированного и оставшегося в вытекшем из адсорбера растворе определяется подобным же образом.

Величина $\rho_{\ni}$, которая соответствует точке Rна кривой 1 (рис. 1), определяет разрешенную нормативными документами максимальную концентрацию поллютанта в вытекающем из адсорбера растворе.

Более общий случай вида зависимости $\rho_{\tau, \mathrm{i}}=f\left(\tau_{\mathrm{i}}\right)$ передает кривая 2 (рис. 1). Дальнейшее усложнение вида кинетической кривой сорбции ничего существенно не изменит.

Количество сорбированного вещества $(N)$ за определенный промежуток времени от $\tau_{1}$ до $\tau_{2}$ может быть определено из зависимости:

$$
N=C_{0} V \int_{\tau_{i}}^{\tau_{i+1}} d(1-\rho(\tau))
$$

где $C_{0}$ - исходная концентрация сорбата, моль/л, а $V$ - объем раствора, прошедшего через адсорбер за рассматриваемый интервал времени. При интегрировании уравнения (3) имеем:

$$
N=-C_{0} V\left(\rho_{\tau_{i+1}}-\rho_{\tau_{i}}\right) .
$$

При условии

$$
\rho_{\tau_{i+1}} \leq \rho_{\tau_{i}},
$$

величина $N$ может быть только положительной и/или равной нулю, что соответствует ее физическому смыслу. В последнем случае адсорбция отсутствует.

Уравнение (4) позволяет оценить количество вещества, оставшегося в растворе в каждом временном подинтервале после выхода его из адсорбера.

Тогда с учетом уравнения (4) количество вещества загрязнителя, сорбированного в каждом временном подинтервале, показанном на рис. 1, может быть рассчитано из зависимостей:

$$
\begin{gathered}
N_{\mathrm{I}}=C_{0} V\left(1-\rho_{\mathrm{A}}+\rho_{\mathrm{B}}\right) ; N_{\mathrm{II}}=C_{0} V\left(1-\rho_{\mathrm{B}}+\rho_{\mathrm{C}}\right) ; \\
N_{\mathrm{III}}=C_{0} V\left(1-\rho_{\mathrm{C}}+\rho_{\mathrm{D}}\right) ; N_{\mathrm{IV}}=C_{0} V\left(1-\rho_{\mathrm{D}}+\rho_{\mathrm{E}}\right) ; \\
N_{\mathrm{V}}=C_{0} V\left(1-\rho_{\mathrm{E}}+\rho_{\mathrm{F}}\right) ; N_{\mathrm{VI}}=C_{0} V\left(1-\rho_{\mathrm{F}}+\rho_{\mathrm{L}}\right) ; \\
N_{\mathrm{VII}}=C_{0} V\left(1-\rho_{\mathrm{L}}+\rho_{\mathrm{M}}\right) .
\end{gathered}
$$

Суммарно с учетом $\Sigma N_{\mathrm{i}}$ имеем:

$$
N_{\Sigma}=C_{0} V\left(7-\rho_{\mathrm{A}}+\rho_{\mathrm{M}}\right) .
$$

В общем случае, уравнение (5) трансформируется в (6):

$$
N_{\Sigma}=C_{0} V\left(w-\rho_{\text {нач }}+\rho_{\mathrm{w}+1}\right),
$$

где $w$ - число рассматриваемых временных интервалов, соответствующих изотерме адсорбции (рис. 1), $\rho_{\text {нач }}$ и $\rho_{\mathrm{w}+1}-$ коэффициенты сорбции соответственно при $\tau$ в начальный и конечный рассматриваемые моменты сорбции.

Если $\rho_{\mathrm{w}+1}=0$, то уравнение (6) принимает вид:

$$
N_{\Sigma}=C_{0} V\left(w-\rho_{\text {нач }}\right) .
$$

Отметим, что интервал с полным удалением сорбата (интервал I, рис. 1) может отсутствовать. Если же он имеет место, то для него $\rho_{\text {кон }}$ также равно 1, и уравнение (7) трансформируется в (2), так как в этом случае $w=1$.

Удельная динамическая емкость сорбента по поллютанту в первом временном подинтервале равна ( $m$ - масса сорбента):

$$
Q_{\mathrm{I}}=\frac{C_{0} V}{m}\left(1-\rho_{\mathrm{A}}+\rho_{\mathrm{B}}\right) .
$$

Суммарная удельная динамическая емкость определяется выражением:

$$
Q_{\Sigma}=\frac{C_{0} V}{m}\left(\mathrm{w}-\rho_{\mathrm{A}}+\rho_{\mathrm{w}+1}\right) .
$$

Если величина прошедшего через адсорбер объема раствора $V$ в рассматриваемых временных подинтервалах не является постоянной, то необходимо учитывать объемы раствора, вытекающие в течение каждого подинтервала, так как $V \neq$ const.To же касается различной протяженности временных подинтервалов, независимо от того является величина $V$ постоянной или нет.

\section{ТЕОРЕТИЧЕСКАЯ ИНТЕРПРЕТАЦИЯ МЕТОДА}

Методика базируется на использовании $\beta$-сплайновых кривых и основана на свойствах геометрической непрерывности и гладкости используемых решений задач геометрического моделирования посредством кубических многочленов [23, 24]. По заданному массиву экспериментальных точек с координатами $\rho_{\mathrm{i}}$ и $\tau_{\mathrm{i}}$ (табл. 1) строится кривая, проходящая через все точки (задача интерполяции). При этом применение составных $\beta$-сплайновых кривых основывается на свойстве геометрической непрерывности и гладкости. В построении составной регулярной кривой важную роль играют условие сопряжения в точках контакта сглаживающих ее отрезков регулярных кривых.

Массив точек, полученных экспериментальным путем и формирующих зависимость $\rho$ от $\tau$ для катионов $\mathrm{Cu}(\mathrm{II})$, и график кубического сплайна (или интерполяционная кривая) при сорбции катионов меди (II) глауконитом, приведены на рис. 2. 
Таблица 1. Уравнения интерполяционной кривой при сорбции катионов $\mathrm{Cu}(\mathrm{II})$

[Table 1. Equations of the interpolation curve for the sorption of $\mathrm{Cu}$ (II) cations]

\begin{tabular}{|c|c|}
\hline $\begin{array}{c}\text { № } \Pi / \text { п } \\
{[\text { No.] }}\end{array}$ & $\begin{array}{c}\text { Уравнение интерполяционной кривой } \\
\text { [Equation of interpolation curve] }\end{array}$ \\
\hline 1 & $\rho=\left(0.0229 \tau^{3}-0.4791 \tau^{2}\right) \cdot 10^{-3}+1$ \\
\hline 2 & $\rho=\left(-0.0287(\tau-10)^{3}+0.2083(\tau-10)^{2}-2.7085(\tau-10)\right) \cdot 10^{-3}+0.975$ \\
\hline 3 & $\rho=\left(0.0271(\tau-20)^{3}-0.6541(\tau-20)^{2}-7.1662(\tau-20)\right) \cdot 10^{-3}+0.94$ \\
\hline 4 & $\rho=\left(-0.0345(\tau-30)^{3}+0.158(\tau-30)^{2}-12.13(\tau-30)\right) \cdot 10^{-3}+0.83$ \\
\hline 5 & $\rho=\left(0.0411(\tau-40)^{3}-0.8779(\tau-40)^{2}-19.326(\tau-40)\right) \cdot 10^{-3}+0.69$ \\
\hline 6 & $\rho=\left(-0.0567(\tau-50)^{3}+0.3537(\tau-50)^{2}-24.5684(\tau-50)\right) \cdot 10^{-3}+0.45$ \\
\hline 7 & $\rho=\left(3.6297(\tau-60)^{3}-13.4688(\tau-60)^{2}-345.002(\tau-60)\right) \cdot 10^{-4}+0.183$ \\
\hline
\end{tabular}

\section{ЭКСПЕРИМЕНТ}

Сорбент предварительно в течение 20 минут обрабатывали 0.1М раствором $\mathrm{NaOH}($ соотношение по массе глауконит/раствор составляло 1:20), затем отмывали от щелочи дистиллированной водой и далее проводили ту же процедуру в $1.0 \mathrm{M} \mathrm{HCl}$ с последующей отмывкой от кислоты. Затем в течение одного часа сорбент переводили в $\mathrm{Na}$-форму в 3M растворе $\mathrm{NaCl}$ и вновь отмывали от ионов хлора. Использована вода с отрицательной реакцией на катионы $\mathrm{Ca}^{2+}$ и $\mathrm{Mg}^{2+}$, анионы хлора и $\mathrm{SO}_{4}{ }^{2-}$.

Исследования проведены при комнатной температуре в специальном адсорбере. Высота слоя сорбента составляла 1.5 см. Линейная скорость подачи раствора через адсорбер v поддерживалась постоянной в пределах0.3 и 0.5 м/ч. Стандартные растворы, содержащие $\mathrm{Cu}(\mathrm{II}), \mathrm{Ni}(\mathrm{II})$ и $\mathrm{Zn}(\mathrm{II})$,готовили с использованием солей $\mathrm{Cu}\left(\mathrm{NO}_{3}\right)_{2} \cdot 3 \mathrm{H}_{2} \mathrm{O}, \mathrm{Ni}\left(\mathrm{NO}_{3}\right)_{2} \cdot 6 \mathrm{H}_{2} \mathrm{O}$, $\mathrm{Zn}\left(\mathrm{NO}_{3}\right)_{2} \cdot 2 \mathrm{H}_{2} \mathrm{O}$ квалификации «ч.д.а».

Содержание катионов в растворах оценивали методом рентгенофлуоресцентной спектроскопии, основанном на концентрировании на целлюлозных ДЭТАТА-фильтрах. При этом проводилось измерение вторичного рентгеновского излучения, возбуждаемого первичным излучением рентгеновской трубки и испускаемого атомами металла. Чувствительность анализа 0.01 мг/л, абсолютная погрешность при доверительной вероятности 0.95 - до 0.0012 мг/л. Использован спектрометр «Спетроскан МАКС-GV».

Глубину сорбции катионов оценивали при комнатной температуре посредством коэффициента $\rho$, определенного ранее. Исходная концентрация катионов (ммоль/л) составляла: $\mathrm{Cu}(\mathrm{II})-0.048$; $\mathrm{Ni}(\mathrm{II})$ - 0.056; Zn(II) - 0.046, что превышало пре-

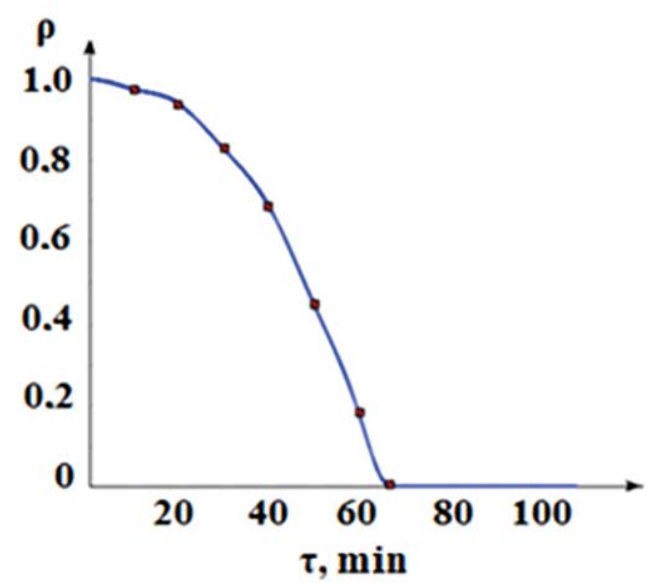

Рис. 2. Кубический сплайн, характеризующий сорбцию катионов $\mathrm{Cu}(\mathrm{II})$

[Fig. 2. Cubic spline characterizing the sorption of $\mathrm{Cu}$ (II) cations]

дельно-допустимую в воде хозяйственно-питьевого и культурно-бытового назначения для $\mathrm{Cu}(\mathrm{II})$ в 3 раза, Ni(II) - почти в 30 раз, для $\mathrm{Zn}(\mathrm{II})$ - почти в 3 раза [25].

\section{ОБСУЖДЕНИЕ РЕЗУЛЬТАТОВ}

Сорбция из проточного раствора при линейной скорости потока $0.3 \mathrm{M} / \textrm{ }$

Координаты экспериментальных точек, полученных при сорбции катионов $\mathrm{Ni}(\mathrm{II})$ и $\mathrm{Zn}(\mathrm{II})$, и вид кубических $\beta$-сплайновых кривых представлены на рис. 3.

Сорбция из проточного раствора при линейной скорости потока $0.5 \mathrm{~m} / \textrm{ }$

Координаты экспериментальных точек используемых катионов и вид кубических сплайнов при их сорбции представлены на рис. 4. 


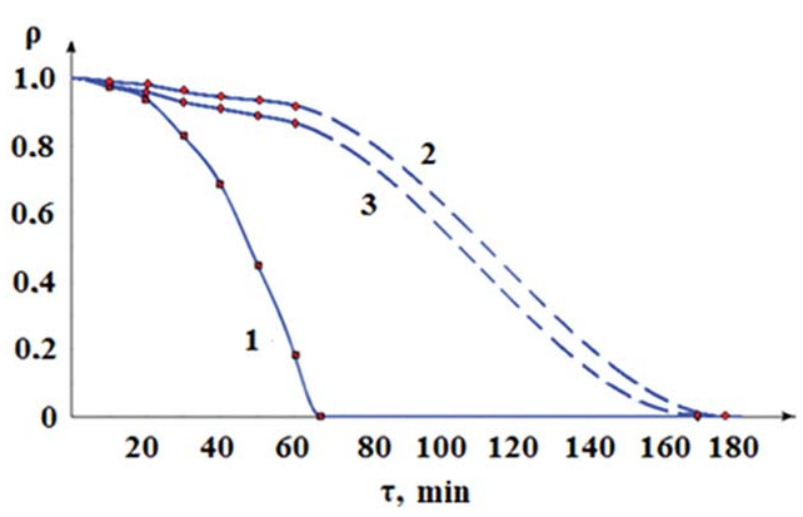

Рис. 3. Вид кубических сплайнов при сорбции катионов: $1-\mathrm{Cu}(\mathrm{II}), 2-\mathrm{Ni}(\mathrm{II}), 3-\mathrm{Zn}(\mathrm{II}) . v=0.3 \mathrm{м} / \mathrm{ч}$

[Fig. 3. Type of cubic splines during the sorption of cations: $1-\mathrm{Cu}$ (II), $2-\mathrm{Ni}(\mathrm{II}), 3-\mathrm{Zn}$ (II). v $=0.3 \mathrm{~m} / \mathrm{h}$ ]

Показанные пунктиром участки кубических сплайнов (рис. 2, 3 и 4), представляют приблизительные данные, поскольку расчеты $\rho_{\mathrm{i}}$ на них не проводились.

По представленным экспериментальным данным с использованием уравнений типа (7) и (8) рассчитаны величины $Q_{\mathrm{i}}$ сорбируемых на глауконите катионов (табл. 2).

Представленные в табл. 2 результаты показывают, что за небольшим исключением в рассматриваемых временных интервалах в первые 60 минут сорбции величина $Q$ для всех катионов изменяется мало. Этот факт является достаточно неожиданным, так как элементарный акт сорбции можно представить как реакцию взаимодействия активного центра (АЦ) с катионом металла:

$$
\mathrm{AЦ}+\mathrm{Me}(\mathrm{II})_{\text {раствор }} \rightarrow \mathrm{Me}(\mathrm{II})_{\text {адс }} .
$$

Тогда по мере занятия АЦ их поверхностная концентрация во времени должна снижаться, а динамическая емкость уменьшаться.

Близость $Q$ катионов в разных временных интервалах, в первом приближении, можно объяс-

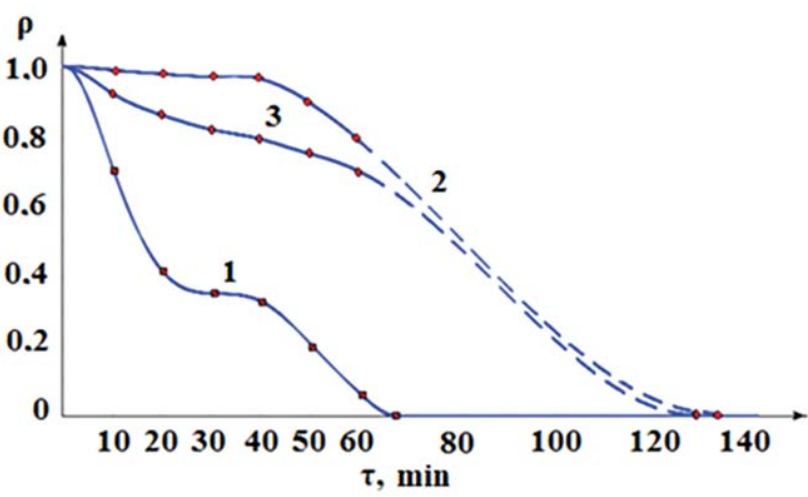

Рис. 4. Вид кубических сплайнов при сорбции катионов: $1-\mathrm{Cu}(\mathrm{II}), 2-\mathrm{Ni}(\mathrm{II}), 3-\mathrm{Zn}(\mathrm{II}) . \mathrm{v}=0.5 \mathrm{M} / \mathrm{ч}$

[Fig. 4. Type of cubic splines during the sorption of cations: $1-\mathrm{Cu}$ (II), $2-\mathrm{Ni}(\mathrm{II}), 3-\mathrm{Zn}$ (II). $v=0.5 \mathrm{~m} / \mathrm{h}$ ]

нить энергетической неравноценностью активных центров. На первой стадии занимаются наиболее активные центры, во втором временном интервале - менее активные, а в третьем - с еще меньшей энергией адсорбции. Причем концентрация АЦ с разной $\Delta G_{\text {адс }}$ примерно одинакова.

С ростом линейной скорости подачи раствора сорбата повышается динамическая емкость по всем рассматриваемым катионам. Подвод ионов может контролироваться внешней или внутренней диффузией [26]. В первом случае возникает разность концентраций катионов в объеме раствора и в адсорбционном слое, которая является движущей силой диффузионного массопереноса.

Рост скорости потока обусловливает снижение этой разности, в силу чего удельная динамическая емкость возрастает, что согласуется с экспериментом, согласно которому величина $\gamma_{4 \text { 'средн }}$ близка к 1.67 , т.е. к отношению линейных скоростей потока.

Время контакта сорбента с сорбатом играет второстепенную роль. Именно этим объясняется тот факт, что доля сорбирующихся катионов $\rho_{i}$ уже

Таблица 2. Влияние природы катиона, линейной скорости потока на динамическую емкость концентрата глауконита

[Table 2. Influence of cation nature, linear flow rate on the dynamic capacity of glauconite concentrate]

\begin{tabular}{|c|c|c|c|c|c|c|}
\hline \multirow{2}{*}{$\begin{array}{c}\tau \text { от начала сорбции, } \\
\text { мин } \\
{[\tau \text { from the beginning of }} \\
\text { sorption, min] }\end{array}$} & \multicolumn{3}{|c|}{$\begin{array}{l}Q \cdot 10^{4} \text { ммоль } / \Gamma \text { при } v=0.3 \mathrm{~m} / \mathrm{ч} \\
{\left[Q \cdot 10^{4} \mathrm{mmol} / \mathrm{g} \text { at } v=0.3 \mathrm{~m} / \mathrm{h}\right]}\end{array}$} & \multicolumn{3}{|c|}{$\begin{array}{l}Q \cdot 10^{4} \text { ммоль } / \Gamma \text { при } v=0.5 \mathrm{~m} / \mathrm{ч} \\
{\left[Q \cdot 10^{4} \mathrm{mmol} / \mathrm{g} \text { at } \mathrm{v}=0.5 \mathrm{~m} / \mathrm{h}\right]}\end{array}$} \\
\hline & $\mathrm{Cu}(\mathrm{II})$ & $\mathrm{Ni}(\mathrm{II})$ & $\mathrm{Zn}(\mathrm{II})$ & $\mathrm{Cu}(\mathrm{II})$ & $\mathrm{Ni}(\mathrm{II})$ & $\mathrm{Zn}(\mathrm{II})$ \\
\hline $0-20$ & 2.6 & 3.1 & 2.4 & 1.8 & 5.0 & 3.6 \\
\hline $20-40$ & 2.0 & 3.1 & 2.2 & 4.1 & 5.0 & 3.9 \\
\hline $40-60$ & 1.3 & 3.1 & 2.4 & 3.2 & 4.3 & 3.9 \\
\hline$Q_{\Sigma}$ & 5.9 & 9.3 & 7.0 & 9.1 & 14.3 & 11.4 \\
\hline
\end{tabular}


в первые 10 минут сорбции достигает $98-99 \%$ (рис. 3 и 4) и редко составляет 70\%. Этим же объясняется и то, что для $\mathrm{Ni}(\mathrm{II})$ величина $Q_{i}$ за 60 минутсущественно возрастает с повышением исходной концентрации сорбата. В период, когда доля занятых активных центров далека от насыщения, повышение $\mathrm{C}_{0}(\mathrm{Ni}(\mathrm{II}))$ с 0.056 до 0.1 ммоль/л увеличивает $Q_{\mathrm{i}}(\mathrm{Ni}(\mathrm{II}))$ в $2.5-3.0$ раза.

Относительную адсорбционную способностьглауконита по отношению к катионам в разные промежутки времени от начала процесса оценивали посредством следующих коэффициентов $\gamma_{i}$ :

$$
\begin{gathered}
\gamma_{1}=Q_{i}(\mathrm{Ni}(\mathrm{II})) / Q_{i}(\mathrm{Cu}(\mathrm{II})), \gamma_{2}=Q_{i}(\mathrm{Zn}(\mathrm{II})) / Q_{i}(\mathrm{Cu}(\mathrm{II})), \\
\gamma_{3}=Q_{i}(\mathrm{Zn}(\mathrm{II})) / Q_{i}(\mathrm{Ni}(\mathrm{II})) .
\end{gathered}
$$

Количественные данные по влиянию на $Q$ линейной скорости подачи раствора получены посредством коэффициента $\gamma_{4}=Q_{i}(\mathrm{Me}(\mathrm{II}))_{0.5} / Q_{i}(\mathrm{Me}(\mathrm{II}))_{0.3}$, где цифра в нижнем индексе характеризует величину линейной скорости потока. Значение $\gamma_{i}>1$ показывает, что катионы металла, указанного в числителе коэффициентов $\gamma_{1}, \gamma_{2}$ и $\gamma_{3}$, сорбируются на глауконите эффективнее приведенного в знаменателе; $\gamma_{\mathrm{i}} \approx 1$ позволяет считать, что сорбционная способность катионов практически близка. При $\gamma_{i}<1$ лучше сорбируются катионы, указанные в знаменателе.

В случае $\gamma_{4}>1$ удельная динамическая емкость сорбента возрастает с повышением линейной скорости потока, при $\gamma_{4} \approx 1-$ не зависит от нее и при $\gamma_{4}<1$ снижается с ростом величины $v$. Соответствующие данные представлены в табл. 3.

Установлено, что катионы $\mathrm{Ni}(\mathrm{II})$ сорбируются глауконитом более эффективно, чем $\mathrm{Cu}(\mathrm{II}), \mathrm{a} \mathrm{Zn}(\mathrm{II})$ занимает промежуточное положение. Особенно четко это следует из величины $\gamma_{1}$ 'средн и $\gamma_{3 \text { ' средн }}$.

Различия в сорбционной способности глауконита по отношению к исследуемым катионам не удается объяснить за счет величин радиусов атомов и ионов, их электроотрицательности, значений первого потенциала ионизации и ионного потенциала, сродства к электрону, изменению свободной энергии и энтропии гидратации $[3,14,16$, 22]. В первом приближении, это обусловлено различиями во влиянии катионов на реорганизацию растворителя (РР) [27].

Имеется в виду, что РР представляет собой перестройку поверхностных состояний из-за квантово-механических флуктуаций, что создает различные условия для взаимодействия катионов с твердой фазой [27].

Уравнения интерполяционных кривых (табл. 2 и 3 ) позволяют оценить $\rho_{i}$ к любому моменту времени. Пусть требуется оценить $\rho_{i}(\mathrm{Ni}(\mathrm{II}))$ через 45 минут от начала сорбции при $v$, равных 0.3 и 0.5 м/ч. Соответствующие уравнения, характерные для временного интервала 40-50 мин, имеют вид :

$$
\begin{aligned}
\rho_{0,3} & =\left(\begin{array}{c}
-0.0009(\tau-40)^{3}+ \\
+0.0239(\tau-40)^{2}- \\
-1.1457(\tau-40)
\end{array}\right) \cdot 10^{-3}+0.945, \\
\rho_{0,5} & =\left(\begin{array}{c}
0.009(\tau-40)^{3}- \\
-0.4318(\tau-40)^{2}- \\
-3.0835(\tau-40)
\end{array}\right) \cdot 10^{-3}+0.965 .
\end{aligned}
$$

Подстановка $\tau=45$ дает результаты $\rho_{0.3}=0.940$ и $\rho_{0.5}=0.940$.

Зная рассчитанные величины $\rho_{i}$, можно с использованием уравнений (8) и (9) оценить соответствующие величины $Q_{\mathrm{i}}$.

С целью выявления селективности глауконита по отношению к катионам металлов были проведены исследования в смешанных растворах следующего состава: 0.125 ммоль/л Са(II) + 0.125 ммоль/л

Таблица 3. Зависимость коэффициентов $\gamma_{i}$ от природы катионов, величины линейной скорости потока и временного интервала сорбции от начала процесса. Числитель - линейная скорость потока равна 0.3 м/ч, знаменатель $-0.5 \mathrm{~m} / \mathrm{\varphi}$

[Table 3. Dependence of the coefficients $\gamma_{i}$ on the nature of cations, the magnitude of the linear flow rate and the time interval of sorption from the beginning of the process. The numerator - the linear flow rate is $0.3 \mathrm{~m} / \mathrm{h}$, the denominator is $0.5 \mathrm{~m} / \mathrm{h}$ ]

\begin{tabular}{|c|c|c|c|c|c|c|}
\hline $\begin{array}{c}\tau \text { от начала сорбции, мин } \\
{[\tau \text { from the beginning of sorption, } \min ]}\end{array}$ & $\gamma_{1}$ & $\gamma_{2}$ & $\gamma_{3}$ & \multicolumn{3}{|c|}{$\gamma_{4}$} \\
\hline $0-20$ & $1.19 / 2.78$ & $0.92 / 2.00$ & $0.77 / 0.78$ & 0.69 & 1.61 & 1.50 \\
\hline $20-40$ & $1.55 / 1.22$ & $1.10 / 0.95$ & $0.71 / 0.78$ & 2.05 & 1.61 & 1.77 \\
\hline $40-60$ & $2.38 / 1.34$ & $1.85 / 1.22$ & $0.77 / 0.91$ & 2.46 & 1.39 & 1.63 \\
\hline $\begin{array}{c}\gamma_{\text {средн }} \\
{\left[\gamma_{\text {average }}\right]}\end{array}$ & $1.77 / 1.78$ & $1.29 / 1.39$ & $0.75 / 0.82$ & 1.73 & 1.54 & 1.63 \\
\hline
\end{tabular}


$\mathrm{Mg}(\mathrm{II})+0.0017$ ммоль/л $\mathrm{Ni}(\mathrm{II})$ с линейной скоростью потока $0.3 \mathrm{~m} /$ ч при высоте слоя сорбента 1.5 см. Указанное содержание Ni(II) - предельно допустимая концентрация катионов в воде хозяйственно-бытового назначения. В этом случае суммарная исходная концентрация $\mathrm{Ca}(\mathrm{II})+\mathrm{Mg}(\mathrm{II})$ превосходила $\mathrm{Ni}(\mathrm{II})$ почти в 150 раз. Можно было ожидать, что в случае, когда все катионы сорбируются на единых активных центрах (АЦ), сорбция $\mathrm{Ni}(\mathrm{II})$ будет существенно подавлена. Если же имеет место селективность АЦ по катионам различной природы, то $\mathrm{Ni}$ (II) будет сорбироваться эффективно. Было установлено, что катионы $\mathrm{Ni}(\mathrm{II})$ сорбируются полностью в течение первых 80 минут процесса, что указывает на высокую селективность активных центров глауконита. При этом присутствие $\mathrm{Ni}$ (II) практически не сказывалось на процессе сорбции $\mathrm{Ca}(\mathrm{II})$ и $\mathrm{Mg}(\mathrm{II})$, которые сорбировались на $97 \% \mathrm{Ca}(\mathrm{II})$ и $98 \% \mathrm{Mg}(\mathrm{II})$ в первые 10 минут процесса.

\section{ЗАКЛЮЧЕНИЕ}

Предложен способ расчета величин динамической емкости сорбента при ламинарном движении раствора через адсорбер в любой момент времени от начала сорбции. Подход, основанный на использовании $\beta$-сплайновых кривых, может быть использован для оценки динамической емкости сорбентов в растворах, содержащих один или несколько катионов, независимо от их природы.

Изучение в качестве сорбатов двухзарядных катионов меди, никеля и цинка показало, что динамическая емкость при линейной скорости подачи растворов $\mathrm{Cu}(\mathrm{II}), \mathrm{Ni}(\mathrm{II})$ и $\mathrm{Zn}(\mathrm{II}) 0.3$ м/ч за первые 60 минут сорбции на глауконите составляет соответственно $5.9 \cdot 10^{-4}, 9.3 \cdot 10^{-4}$ и $7.0 \cdot 10^{-4}$ ммоль/г и возрастает при $v=0.5$ м/ч пропорционально повышению линейной скорости потока.

Относительная сорбционная способность глауконита по отношению к изученным катионамизменяется в соответствии с рядом:

$$
\mathrm{Ni}(\mathrm{II})>\mathrm{Zn}(\mathrm{II})>\mathrm{Cu}(\mathrm{II}) \text {. }
$$

Специальными экспериментами в присутствии катионов $\mathrm{Ca}(\mathrm{II}), \mathrm{Mg}(\mathrm{II})$ и $\mathrm{Ni}(\mathrm{II})$, когда суммарное содержание первых двух почти в 150 раз превышает содержание никеля, показано, что катионы Ni(II) избирательно сорбируются на активных центрах, на которых сорбция $\mathrm{Ca}(\mathrm{II})$ и $\mathrm{Mg}(\mathrm{II})$ не имеет места.

Экспериментальные результаты получены на оборудовании Центра коллективного пользова- ния научным оборудованием ТГУ имени Г. Р. Державина.

\section{СПИСОК ЛИТЕРАТУРЫ}

1. Srivastava P.,Singh B., Angove M. // J. Coll. Interface Sci., 2005,vol. 290, № 1, pp. 28-38. DOI: 10.1016/j. jcis.2005.04.036

2. Singh K. K., Rastogy R., Hasan S. H. // J. Coll. Interface Sci., 2005, vol. 290, № 1, pp. 61-68. DOI: 10.1016/ j.jcis.2005.04.011

3. Egirany D.E., Baker A.R., Andrews J.E. // J. Coll. Interface Sci., 2005, vol. 291, № 2, pp. 319-325. DOI: 10.1016/j.jcis.2005.05.007

4. Zhao J., Zhy Y. J., Wu J., Zheng J.-O., Zhao X.-Yu., Lu B.-Q., Chen F. // J. Coll. Interface Sci., 2014, vol. 418, № 1, pp. 208-215. DOI: 10.1016/j.jcis.2013.12.016

5. Teutli-Sequeira A., Solache-Ríos M., Martínez-MirandaV., Linares-Hernández I. // J. Coll. Interface Sci., 2014, vol. 418, № 1, pp. 254-260. DOI: 10.1016/j.jcis.2013. 12.020

6. Liu B., Lu J., Xie Yu., Yang B. Wang X., Sun R. // J. Coll. Interface Sci., 2014, vol. 418, № 1, pp. 311-316. DOI: 10.1016/j.jcis.2013.12.035

7. Конькова Т. В., Алехина М. Б., Михайличенко А. И., Канделаки Г. И., Морозов А. Н. // Физикохимия поверхности и защита материалов, 2014, т. 50, № 3, c. 277-281. DOI: $10.7868 / \mathrm{S} 0044185614030085$

8. Дударева Г. Н., Петухова Г. Н., Нгуен А. Т. Н., Сырых Ю. С. // Физикохимия поверхности и защита материалов, 2013, т. 49, № 4, с. 389-396. DOI: 10.7868/ S0044185613040025

9. Огородова Л. П., Мельчакова Л. В., Вигасина М. Ф., Крупская В. В., Киселева И. А. // Журнал физическойхимии, 2014, т. 88, № 11, с. 1824-1827. DOI: $10.7868 / \mathrm{S} 0044453714100306$

10. Бельчинская Л. И., Ходосова Н. А., Стрельникова О. Ю., Петухова Г. А., Ciganda L. // Физикохимия поверхности и защита материалов, 2015, т. 51, № 5, c. 487-494. DOI: $10.7868 / \mathrm{S} 0044185615050046$

11. Котова Д. Л., Васильева С. Ю., Крысанова Т. А., Хромова А. С., Фам Тхи Гам // Физикохимия поверхности и зашита материалов, 2015, т. 51, № 4, с. 351-356. DOI: $10.7868 / \mathrm{S} 0044185615040191$

12. Помазкина О. И., Филатова Е. Г., Пожидаев Ю. Н. // Физикохимия поверхности и защита материалов, 2015 , т. 51, № 4, с. 370-374. DOI: $10.7868 /$ S0044185615040269

13. Костин А. В., Мостальгина Л. В., Бухтояров О. И. // Физикохимия поверхности и защита материалов, 2015 , т. 51, № 5, с. 477-482. DOI: $10.7868 /$ S0044185615050174

14. Vieira M. G. A., Almeida Neto A.F., Gimenes M. L., M. G. C. da Silva // J. of Hazardous Materials, 2010, vol. 176, №1, pp. 109-118. DOI: 10.1016/j.jhazmat. 2009.10.128

15. Дударева Г. Н., Рандин О. И., Петухова Г. А., Вакульская Т. И. // Физикохимия поверхности и защита 
материалов, 2015, т. 51, № 6, с. 582-586. DOI: 10.7868/ S0044185615060066

16. Liu Zhi-rong, Zhou Shao-qi // Process Safety and Environmental Protection, 2010, vol. 88, № 1, pp. 62-66. DOI: 10.1016/j.psep.2009.09.001

17. Ozlem Korkut, Enes Sayan, Oral Lacin, Bahar Bayrak // Desalination, 2010, vol. 259, № 3, pp. 243-248. DOI: 10.1016/j.desal.2010.03.045

18. Abollino O., Aceto M., Malandrino M., Sarzanini C., Mentasti E. // Water Research, 2003, vol. 37, pp. 1619-1627. DOI: 10.1016/S0043-1354(02)00524-9

19. Vieira M. G. A., Almeida Neto A. F., Grimens M. L., Silva M. G. C. //Hazardous Materials, 2010, vol. 176, № 2, pp. 109-118. DOI: 10.1016/j.jhazmat.2009.10.128

20. Almeida Neto A. F., Vieira M. G. A.,Silva M. G. C. // J. Water process engineering, 2014, vol. 3, № 1, pp. 90-97. DOI: $10.1016 /$ j.jwpe.2014.05.014

21. Gonzalez A. G., Pokrovsky O. S. // J. Coll. Interface Sci., 2014, vol. 415, pp. 169-178. DOI: 10.1016/j. jcis.2013.10.028
22. Вигдорович В. И., Цыганкова Л. Е., Шель Н. В., Есина М. Н., Шель Е. Ю., Омутков М. С., Пустынников Я. А. Материаль VIII Международной научно-инновационной молодежной конференции «Современные твердофазные технологии: теория, практика, инноваиионный менеджмент». Тамбов, Изд-во Чеснокова А. В., 2016,c. 34-49.

23. Самарский А. Н. Введение в численные методы. М.: Наука, Главная редакция физико-математической литературы, 1982, 272 с.

24. Шикин Е. В., Боресков А. В. Компьютерная графика. Динамика, реалистические изображения. М.: Диалог - МИФИ, 1995, 288 с.

25. Вишняков Я. Д., Бурцева Н. Н., Киселева С. П., Рыков С. В., Рязанова Н. Е. Нормирование и снижение загрязнения окружающей среды. М.: Академия, 2015, $386 \mathrm{c}$.

26. ВенициановЕ. В., Рубинштейн Р. Н. Динамика сорбции из жидких сред. М.: Наука, 1983, 253 с.

27. Kuznetsov A. M. Charge Transfer in Chemical Reaction Kinetics. Presses Polytechniqiueset Universitaires Romandes, Lausanne, 1997, 110 p.

\title{
GRAPHOANALYTICAL ESTIMATE OF THE DYNAMIC CAPACITY OF GLAUCONITE IN THE JOINT SORPTION OF THE Cu (II), Ni (II), Zn (II)Cu(II), Ni(II), Zn(II) CATIONS
}

\author{
(C) 2018 V. I. Vigdorovich ${ }^{1,2}$, T. V. Zhukovskaia², L. E. Tsygankova ${ }^{3}$, M. N. Uryadnikova ${ }^{3}$, N. V. Shel ${ }^{2}$ \\ ${ }^{1}$ All-Russian Research Institute for the Use of Machinery and Petroleum Products in Agriculture, \\ 28 Novo-Rubezhny per., 392022 Tambov, Russia \\ ${ }^{2}$ Tambov State Technical University, 106 Sovetskaya str., Tambov, Russia \\ ${ }^{3}$ Tambov State University named after G. R. Derzhavin, 33 International'nayastr., 392000 Tambov, Russia \\ e-mail: vits21@mail.ru
}

Received 02.10.2017

\begin{abstract}
The paper proposes a calculation method to estimate the amount of adsorbate substance $(N)$ and the dynamic capacity of the sorbent $(Q)$ under the conditions of purification from pollutants of flowing solutions. The considered approaches are valid for using sorbents and removing sorbates of any nature from flowing media, regardless of the volume flow rates of the eluate under laminar flow conditions. The method is based on the use of $\beta$-spline curves and is based on the properties of geometric continuity used in solving problems of geometric modelling by means of cubic polynomials. It allows:

- calculating the values of the dynamic capacity $Q$ at any time from the beginning of the sorption in the absence of an experimental estimate of the sorption coefficient $\rho_{\tau_{\mathrm{i}}}$

- evaluating the calculated time of continuous operation of the sorbent until its replacement or regeneration.

At room temperature, glauconite $Q_{\mathrm{i}}$ values were obtained for $\mathrm{Cu}$ (II), $\mathrm{Ni}$ (II), $\mathrm{Zn}$ (II) cations at joint sorption as a function of the nature of the adsorbate with the account of the height of the adsorbate, the linear flow rate, and the arbitrary time interval from the beginning of the process. The relative sorption capacity of cations depending on the same factors was evaluated. It is shown that as the flow rate increases the dynamic capacity of the sorbent increases in the series:
\end{abstract}

$$
Q_{\mathrm{Ni}(\mathrm{II})}>Q_{\mathrm{Zn}(\mathrm{II})}>Q_{\mathrm{Cu}(\mathrm{II})}
$$

Keywords: glauconite, stream, sorption, copper, nickel, zinc, dynamic capacity, calculation. 


\section{ACKNOWLEDGMENTS}

The results of the experiment were obtained using the equipment of Shared Scientific Equipment Centre of Derzhavin Tambov State University.

\section{REFERENCES}

1. Srivastava P., Singh B., Angove M. J. Coll. Interface Sci., 2005, vol. 290, no. 1, pp. 28-38. DOI: 10.1016/j. jcis. 2005.04 .036

2. Singh K. K., Rastogy R., Hasan S. H. J. Coll. Interface Sci., 2005, vol. 290, no. 1, pp. 61-68. DOI: 10.1016/j. jcis.2005.04.011

3. Egirany D. E., Baker A. R., Andrews J. E. J. Coll. Interface Sci., 2005, vol. 291, no. 2, pp. 319-325. DOI: 10.1016/j.jcis.2005.05.00

4. Zhao J., Zhy Y. J., Wu J., Zheng J.-O., Zhao X.-Yu., Lu B.-Q., Chen F. J. Coll. Interface Sci., 2014, vol. 418, no. 1 , pp. 208-215. DOI: 10.1016/j.jcis.2013.12.016

5. Teutli-Sequeira A., Solache-Ríos M., Martínez-MirandaV., Linares-Hernández I. J. Coll. Interface Sci., 2014, vol. 418, no. 1, pp. 254-260. DOI: 10.1016/j. jcis.2013.12.020

6. Liu B., Lu J., Xie Yu., Yang B. Wang X., Sun R. J. Coll. Interface Sci., 2014, vol. 418, no. 1, pp. 311-316. DOI: 10.1016/j.jcis.2013.12.035

7. Konkova T. V., Alekhina M. B., Mikhaylichenko A. I., Kandelaki G. I., Morozov A. N. Protection of Metals and Physical Chemistry of Surfaces, 2014, vol. 50, no. 3, pp. 77-281. DOI: 10.7868/S0044185614030085

8. Dudareva G. N., Petukhova G. N., Nguyen A. T. N., Syrykh Yu. S. Protection of Metals and Physical Chemistry of Surfaces, 2013, vol. 49, no. 4, pp. 389-396. DOI: 10.7868/ S0044185613040025

9. Ogorodova L. P., Melchakova L. V., Vigasina M. F., Krupskaya V. V., Kiseleva I. A. J. of Physical Chemistry., 2014, vol. 88, no. 11, pp. 1824-1827. DOI: $10.7868 /$ S0044453714100306

10. Belchinskaya L. I., Khodosova N. A., Strelnikova O. Yu., Petukhova G. A., Ciganda L. Protection of Metals and Physical Chemistry of Surfaces, 2015, vol. 51, no. 5, pp. 487-494. DOI: 10.7868/S0044185615050046

11. Kotova D. L., Vasilyeva S. Yu., Krysanova T. A., Khromova A. S., Fam Thi Gam. Protection of Metals and Physical Chemistry of Surfaces, 2015, vol. 51, no. 4, pp. 351-356. DOI: 10.7868/S0044185615040191

12. Pomazkina O. I., Filatova E. G., Pozhidaev Yu. N. Protection of Metals and Physical Chemistry of Surfaces, 2015, vol. 51, no. 4, pp. 370-374. DOI: 10.7868/ S0044185615040269

13. Kostin A. V., Mostagina L. V., Bukhtoyarov O. I. Protection of Metals and Physical Chemistry of Surfaces, 2015, vol. 51, no. 5, pp. 477-482. DOI: 10.7868/ S0044185615050174
14. Vieira M. G. A., Almeida Neto A. F., Gimenes M. L., M. G. C. da Silva. J. of Hazardous Material, 2010, vol. 176, no.1, pp. 109-118. DOI: 10.1016/j.jhazmat.2009.10.128

15. Dudareva G. N., Randin O. I., Petukhova G. A., Vakulskaya T. I. Protection of Metals and Physical Chemistry of Surfaces, 2015, vol. 51, no. 6, pp. 582-586. DOI: 10.7868/S0044185615060066

16. Liu Zhi-rong, Zhou Shao-qi. Process Safety and Environmental Protection, 2010, vol. 88, no. 1, pp. 62-66. DOI: 10.1016/j.psep.2009.09.001

17. Ozlem Korkut, Enes Sayan, Oral Lacin, Bahar Bayrak. Desalination, 2010, vol. 259, no. 3, pp. 243-248. DOI: 10.1016/j.desal.2010.03.045

18. Abollino O., Aceto M., Malandrino M., Sarzanini C., Mentasti E. Water Research, 2003, vol. 37, pp. 1619-1627. DOI: 10.1016/S0043-1354(02)00524-9

19. Vieira M. G. A., Almeida Neto A. F., Grimens M. L., Silva M. G. C. Hazardous Materials, 2010, vol. 176, no. 2, pp. 109-118. DOI: 10.1016/j.jhazmat.2009.10.128

20. Almeida Neto A. F., Vieira M. G. A., Silva M. G. C. J. Water Process Engineering, 2014, vol. 3, no. 1, pp. 90-97. DOI: 10.1016/j.jwpe.2014.05.014

21. Gonzalez A. G., Pokrovsky O. S. J. Coll. Interface Sci., 2014, vol. 415, pp. 169-178. DOI: 10.1016/j. jcis.2013.10.028

22. Vigdorovich V. I., Tsygankova L. E., Shel N. V., Esina M. N., Shel E. Yu., Omutkov M. S., Pustynnikov Ya. A. Materialy VIII Mezhdunarodnoi nauchno-innovatsionnoi molodezhnoi konferentsii «Sovremennye tverdofaznye tekhnologii: teoriya, praktika, innovatsionnyi menedzhment» [Proc. 8th Int. Scientific and Innovative Youth Conference «Modern Solid-Phase Technologies: Theory, Practice, Innovative Management»]. Tambov, Chesnokova A. V. Publ., 2016,pp. 34-49. (in Russ.)

23. Samarskii A. N. Vvedenie v chislennyemetody [Introduction to Numerical Methods]. Moscow, Nauka, Home Edition of Physical and Mathematical Literature Publ., 1982, 272 p. (in Russ.)

24. Shikin E. V., Boreskov A.V. Komp'yuternaya grafika. Dinamika, realisticheskie izobrazheniya [Computer Graphics. Dynamics, Realistic Images]. Moscow, Dialogue - MEPhI. Publ., 1995, 288 p. (In Russ.)

25. VishnyakovYa. D., Burtseva N. N., Kiseleva S. P., Rykov S. V., Ryazanova N. E. Normirovanie i snizhenie zagryazneniya okruzhayushchei sredy [Normalization and Reduction of Environmental Pollution]. Moscow, Academy Publ., 2015, 386 p.

26. Venitsianov E. V., Rubinshtein R. N. Dinamika sorbtsii iz zhidkikh sred [Dynamics of Sorption from Liquid Media]. Moscow, Science Publ., 1983, 253 p.

27. Kuznetsov A. M. Charge Transfer in Chemical Reaction Kinetics. Presses Polytechniqiues et UniversitairesRomandes, Lausanne, 1997, 110 p. 
Вигдорович Владимир Ильич - д. х. Н., профессор, академик РАЕН, заслуженный деятель науки и техники РФ, главный научный сотрудник ВНИИТиН, профессор Тамбовского государственного технического университета по совместительству; тел./факс: +7(4752) 446414, e-mail: vits21@mail.ru

Жуковская Татьяна Владимировна - к. ф.-м. н., доцент кафедры высшей математики, Тамбовский государственный техническийуниверситет; тел.: +7(960) 6674147

Цыганкова Людмила Евгеньевна - д. х. н., професcop, академик РАЕН, зав. кафедрой химии и экологической безопасности, Тамбовский государственный университет имени Г. Р. Державина; тел.: +7(902) 7276258, e-mail: vits21@mail.ru

Урядникова Марина Николаевна - к. х. н., старший преподаватель кафедры химии и экологической безопасности, Тамбовский государственный университет имени Г. Р. Державина; тел.: +7(910) 6567866, e-mail: esinamarisha@rambler.ru

Шель Наталья Владимировна - д. х. н., профессор кафедры «Химия и химические технологии», Тамбовский государственный технический университет; тел.: +7(910) 6549099, e-mail: vits21@mail.ru
Vladimir I. Vigdorovich - Dr. Sci. (Chem.), Professor, Academician of the Russian Academy of Natural Sciences, Honored Worker of Science and Technology of the Russian Federation, Chief Scientific Officer of VNIITiN, Professor of Tambov State Technical University in combination; tel./fax: +7(4752) 446414, e-mail: vits21@mail.ru

Tatiana V. Zhukovskaia - Cand. Sci. (Phys.-Math), Associate Professor of the Higher Mathematics Department of Tambov State Technical University; tel.: +7(960) 6674147

Lyudmila E. Tsygankova - Dr. Sci. (Chem.), Professor, Academician of the Russian Academy of Natural Sciences, Head of the Department of Chemistry and Ecological Safety, Tambov State University named after G.R. Derzhavin; tel.: +7(902) 7276258, e-mail: vits21@mail.ru

Marina N. Uryadnikova - Cand. Sci. (Chem.), Senior Lecturer of the Department of Chemistry and Environmental Safety, Tambov State University named after G. R. Derzhavin; tel.: +7(910) 6567866, e-mail: esinamarisha@, rambler.ru

Natalia V.Shel - Dr. Sci. (Chem.), Professor of the Department «Chemistry and Chemical Technologies», Tambov State Technical University: tel.: +7(910) 6549099, e-mail: vits21@mail.ru 